\title{
Synthesis of High-Yield Gold Nanoplates: Fast Growth Assistant with Binary Surfactants
}

\author{
Changshun Wang, ${ }^{1}$ Caixia Kan, ${ }^{1}$ Jiejun Zhu, ${ }^{2,3}$ Xiaoling Zeng, ${ }^{1}$ Xiangfu Wang, \\ Hongchen Li, ${ }^{1}$ and Daning Shi ${ }^{1}$ \\ ${ }^{1}$ College of Science, Nanjing University of Aeronautics and Astronautics, Nanjing 210016, China \\ ${ }^{2}$ National Laboratory of Solid State Microstructures, Nanjing University, Nanjing 210093, China \\ ${ }^{3}$ Department of Applied Physics, Nanjing University of Technology, Nanjing 210009, China
}

Correspondence should be addressed to Caixia Kan, cxkan@nuaa.edu.cn and Daning Shi, shi@nuaa.edu.cn

Received 4 September 2009; Revised 24 February 2010; Accepted 14 April 2010

Academic Editor: Yong Wang

Copyright (C) 2010 Changshun Wang et al. This is an open access article distributed under the Creative Commons Attribution License, which permits unrestricted use, distribution, and reproduction in any medium, provided the original work is properly cited.

\begin{abstract}
A novel and simple chemical method was developed to fastly synthesize Au nanoplates with size of micrometers and tens of nanometers in thickness. The synthesis was carried out within 30 minutes by thermal reduction of precursors $\left(\mathrm{HAuCl}_{4}\right)$ in the presence of binary surfactants Poly(vinylpyrrolidone) (PVP) and cetyltrimethylammonium bromide (CTAB) in ethylene glycol (EG) solution. The presence and concentration of PVP and CTAB in the growth solution play important roles in the fast formation of $\mathrm{Au}$ nanoplates. The obtained $\mathrm{Au}$ nanoplates exhibit strong surface plasmon absorption in the near-infrared region (NIR), displaying a considerable dependence on the shape and size. Characterizations by Transmission electron microscope and X-ray diffraction indicated that the nanoplates are single crystals with (111) planes as two basal surfaces. Explanations for the nuclei formation and crystal growth behind anisotropic Au nanoplates were proposed.
\end{abstract}

\section{Introduction}

For more than one decade, nanometer-sized noble metal particles, especially gold $(\mathrm{Au})$ and silver $(\mathrm{Ag})$, have attracted considerable attention not only because of their size- and shape-dependent optical and electronic properties distinctly different from their bulk materials, but also due to their potential applications in thermal, catalysis, surface-enhanced Raman scattering, photoelectronic devices, biomedical diagnostics, and other related fields [1-5]. In the case of optical property, it is found that the optical absorption of nanostructures is highly dependent on the exact morphology and the aspect ratios of Au products. Both experiment data and theoretical calculation show that one of the plasma resonances, in particular for Au nanorods is strong and tunable throughout the visible to nearinfrared region in the spectrum, which opens new possibilities for many attractive fields [6-9]. And now it is accepted that nanogold does not glitter, but its future looks bright.
Because of sizes and shapes distribution, most of the presented properties in published works reflect a mean effect of the sample rather than a single nanoparticle. The monodispersity of nanoparticles and the uniformity of size and shape are important for both fundamental research and potential technological applications. At present, although some methods related to colloidal chemistry have been established for the fabrication of shape-controlled $\mathrm{Au}$ and $\mathrm{Ag}$ nanostructures, much attention was paid on developing new methods to control the anisotropic growth of crystals into defined morphologies. For anisotropic $\mathrm{Au}$ and Ag nanostructures, various shapes, such as nanorods, nanowires, nanoplates, nanorings, polyhedron, nanoprisms, and nanocubes [10-25], can be synthesized by seedmediated synthesis $[15,19]$, template-directed synthesis [16], polymer-assisted synthesis [17], microwave heating [18], and chemical approach (electrochemistry, photochemistry, and sonochemistry) $[24,25]$.

Among these anisotropic nanoparticals, the monodispersity of two-dimensional nanoplates with flat surfaces 
and regular shapes has attracted great attention due to their promising applications in distinctive optical properties associated with the collective oscillations of conduction electrons $[21,26]$. In the case of $\mathrm{Au}$ platelets, the crystal was usually synthesized via the modified polyol procedure (ethylene glycol in most cases) with the presence of capping agents. Among the capping agents, Poly(vinylpyrrolidone) (PVP) was extensively used in preparing many types of colloidal nanocrystals, because it not only has a reducing effect on metal ions and but also can prevent the product from agglomeration [27]. Another cationic surfactant, cetyltrimethylammonium bromide (CTAB), was also used to control the morphology of nanoparticles (Au nanorods in most cases) [28-30]. Recently, one group reported a wet-chemical process for the seed-mediated growth of $\mathrm{Au}$ nanoplates on an indium tin oxide surface with binary surfactants PVP and CTAB [31]. At present, the clear action of surfactants during the fabrication of nanoparticles is still a challenge.

In our recent work, we explored a simple and modified time-saving polyol method for mass fabrication of $\mathrm{Au}$ nanoplates. The obtained $\mathrm{Au}$ nanoplates are of singlecrystalline with several micrometers in size and tens of nanometers in thickness. The optical properties and the effect of surfactants concentration on the shape and size were investigated. Growth mechanism for the Au nanoplates was also proposed.

\section{Experimental Section}

2.1. Chemicals and Materials. Cetyltrimethylammonium bromide (CTAB, $\geq 99 \%$, Aldrich) and Poly(vinylpyrrolidone) (PVP, K-30, $\mathrm{Mw}=40000$, Aldrich) were purchased from China Sinopharm Chemical Reagent Co., Ltd. PVP was obtained by using the molecular weight of the polymer reported by the supplier, taking into account the mass of the repeating unit $(111 \mathrm{~g} / \mathrm{mol})$. Ethylene glycol (EG, Aldrich) and hydrogen tetrachloroaurate tetrahydrate $\left(\mathrm{HAuCl}{ }_{4} \cdot 4 \mathrm{H}_{2} \mathrm{O}\right.$, Aldrich) were purchased from Shanghai Chemical Reagent Co., Ltd. All of the materials were obtained as received without further purification. The acetone and ultrapure deionized water $\left(18.3 \mathrm{M} \Omega \cdot \mathrm{cm}^{-1}\right)$ were used for centrifugation.

2.2. Synthesis of $A u$ Nanoplates. In a typical experiment for synthesis of $\mathrm{Au}$ nanoplates, $10 \mathrm{ml} \mathrm{EG}$ was added into the round-bottom flask and heated to $150^{\circ} \mathrm{C}$ in air atmosphere under magnetic stirring in an oil bath. $2 \mathrm{~mL} \mathrm{HAuCl}_{4}$ solution $(0.05 \mathrm{M}$, in $\mathrm{EG})$ was added into $4 \mathrm{~mL}$ CTAB solution $(0.05 \mathrm{M}$, in EG). Then the mixture was kept at $80^{\circ} \mathrm{C}$ in an oven for 10 minutes. After preheating, we injected the mixture quickly into the heated EG solution within 15 seconds. Then $3 \mathrm{~mL}$ PVP solution $(111 \mathrm{mg} / \mathrm{mL}$, in EG) was injected dropwise over a period of $\sim 2$ minutes (the molar concentration ratio (R) between the repeating unit of PVP and Au is 30). The reactant mixture was continuously stirred at $150^{\circ} \mathrm{C}$ up to 30 minutes. For the subsequent characterization, the samples were centrifuged at $\sim 3000 \mathrm{rpm}$ with acetone for 20 minutes.

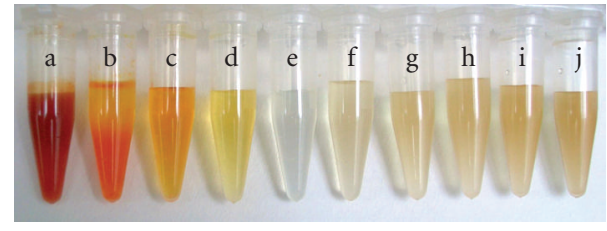

FIGURE 1: The color changes of the reactant mixture during the synthesis of Au nanoplates: (a) the mixed solution of CTAB and $\mathrm{HAuCl}_{4}$ preheated at $80^{\circ} \mathrm{C}$, (b) after the mixture was added into the heated EG solution, (c)-(j) 2, 5, 7, 10, 15, 20, 25, 30 minutes after addition of PVP.

To remove the possible contamination, the product was centrifuged with ultrapure deionized water twice. And the final samples were kept in the ultrapure deionized water.

2.3. Characterization. Optical absorption spectra for the samples were recorded on a UV-vis-NIR spectrophotometer (SP-752PC) in the wavelength range from $200 \mathrm{~nm}$ to $1100 \mathrm{~nm}$. For optical absorption measurement, the samples sampled at different reaction times were dispersed in EG in glass cell with a 5-mm path length. Transmission electron microscopy (TEM), high resolution transmission electron microscopy (HRTEM) images and selected-area electron diffraction (SAED) studies were taken on JEM-1010 and JEM-2100. For TEM measurements, a drop of the sample was dispersed onto a copper grid and evaporated in air at room temperature. Field-emission scanning electron microscopy (FE-SEM) measurements were carried out with a microscope (ESEM, Sirion200, FEG) operated at $20 \mathrm{kV}$. The X-ray diffraction (XRD) pattern was conducted on a diffractometer (Ultima-III, Rigaku) with $\mathrm{Cu} \mathrm{K} \alpha$ radiation. Atomic force microscopy (AFM) images were generated with a multimode scanning probe microscope (SPM) in the tapping AFM mode (Veeco, MultimodeNanoScope 3D).

\section{Results and Discussion}

3.1. Color Changes and the UV-vis-NIR Spectra. Figure 1 shows the color changes of the reactant mixture sampled at different times. The color of the sample turned from yellow to red brown (Figure 1(a)), when the EG solution mixed with $\mathrm{CTAB}$ and $\mathrm{HAuCl}_{4}$ was kept at $80^{\circ} \mathrm{C}$ for 10 minutes. After the mixture was injected into the preheated EG solution, the color changed to yellowish (Figure 1(b)). After addition of PVP solution, the reaction system changed quickly into colorless with reaction time of about 7 minutes (Figure 1(e)). With time increasing, the shiny products could be observed. After about 30 minutes the reaction and crystal growth were completed, which could also be reflected from the optical spectra measurement.

Corresponding to the changes in the solution color, the crystal growth process has also been monitored by UV-vis-NIR spectroscopy. Optical absorption spectra for the samples in EG solution were presented in Figure 2. At the initial stage, only one absorption peak at $340 \mathrm{~nm}$ was detected (Figure 2(a)). Introducing CTAB in the EG 


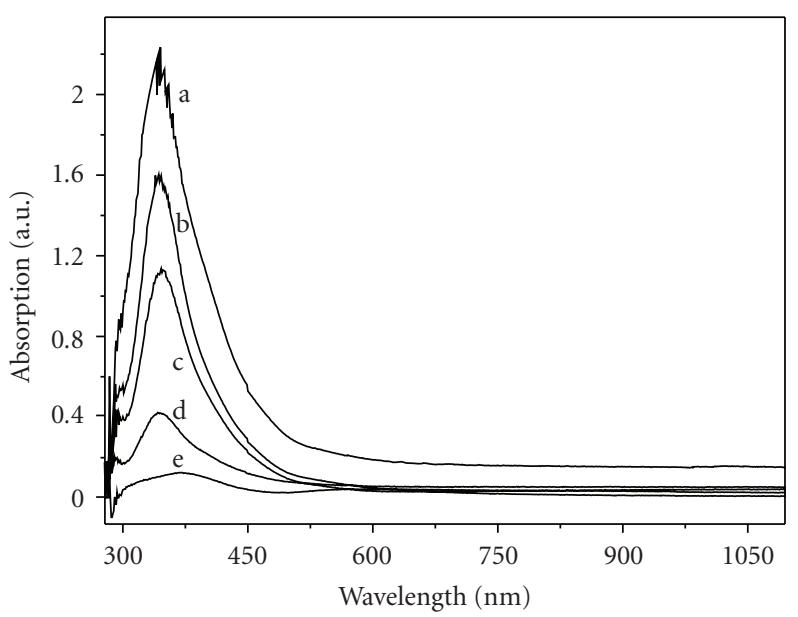

a: The mixtrue was prheated at $80^{\circ} \mathrm{C}$ d: 5 min b: The mixture was added into EG e: $7 \mathrm{~min}$ c: After addition of PVP

(a)

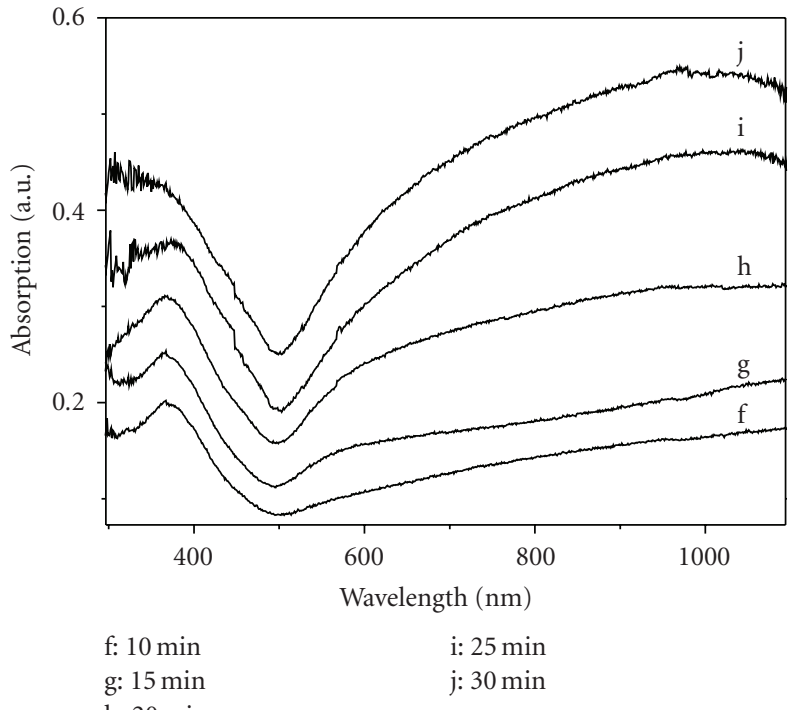

(b)

FIGURE 2: The UV-vis-NIR absorption spectra of the reaction mixture sampled at different times during the synthesis. All the samples were dispersed in EG solution.

solution of $\mathrm{HAuCl}_{4}$, another $\mathrm{Au}$ ion $\left(\mathrm{AuBr}_{4}{ }^{-}\right)$with an absorption spectrum in the UV part was formed [30, 32]. The peak of unique "fingerprint" for $\mathrm{AuCl}_{4}{ }^{-}$ions in aqueous solution at $320 \mathrm{~nm}$ shifted to $340 \mathrm{~nm} \mathrm{[21].} \mathrm{One}$ possibility is the coexistence of $\mathrm{AuBr}_{4}{ }^{-}$and $\mathrm{AuCl}_{4}{ }^{-}$ions. Corresponding to the color changes after addition of PVP, the absorption peak decreased with time and disappeared after 7 minutes (see curve-e in Figure 2(a)), indicating a complete reduction of $\mathrm{Au}(\mathrm{III})$ to $\mathrm{Au}(\mathrm{I})$. Then with the reaction time, the reduction of $\mathrm{Au}(\mathrm{I})$ to atomic $\mathrm{Au}(0)$ results in nucleation of $\mathrm{Au}(0)$ and crystal growth processes in the solution. Subsequently, the absorption in the NIR region ever increased as indicated by the optical absorption spectra (see curve-f in Figure 2(b)). With increase of reaction time, the broad absorption band shifted to $\sim 1050 \mathrm{~nm}$ (see curves $i$ and $j$ ), which is the mean effect of the absorption for both in-plane and out-of-plane polarizations assigned to the dipole and quadrupole plasmon resonances of nanoplates in EG solution (Note: quadrupole resonance of Au nanostructures at $\sim 820 \mathrm{~nm}$ is rarely observed because it was usually overlapped by the broad in-plane dipole resonance) $[13,33,34]$.

3.2. Structural Characterizations. The typical FE-SEM images of Au nanoplates with low- and high-magnification are shown in Figures 3(a) and 3(b). It can be seen that the final product is dominated by regular hexagonal shape with average edge size of $\sim 5 \mu \mathrm{m}$. Some spherical Au nanoparticles are also observed as by-products. AFM was used to delineate the shape and size of the obtained Au nanoplates. The section analysis on AFM image shows that the nanoplate has a quite smooth surface over the width and is about $28 \mathrm{~nm}$ in average thickness (Figures 3(c) and 3(d)).
Figure 4 gives the XRD pattern of the Au nanoplates. Four peaks assigned to (111), (200), (220), and (311) are face-centered cubic $(f c c)$ structure of $\mathrm{Au}$ nanoplates. In contrast to the overwhelming strong (111) diffraction peak at about $38.2^{\circ}$ in the pattern, other diffraction peaks (see insertion) are very weak (The relative diffraction intensity of $(200) /(111)$ is 0.005 , which is much lower than the corresponding conventional value (0.52, JCPDS 04-0784)). The lattice constant calculated from this XRD pattern was $4.080 \AA$, which is perfectly in agreement with the literature value of $4.086 \AA$. The XRD result and above SEM images indicate that the main product is single crystalline with (111) planes as two basal surfaces, and the (111) planes tend to be oriented parallel to the substrate surface. For Au structure of this morphology, it is quite common that the lowest free energy of the (111) planes can induce the formation of platelike structure in chemical method $[35,36]$.

TEM images of the Au nanoplates are shown in Figures 5(a) and 5(b). Figure 5(a) presents some typical Au nanoplates of regular hexagonal shape. The background of carbon capped copper grid can be obviously observed, providing another piece of evidence for that the nanoplates are ultrathin in accordance with the AFM result. The SAED pattern (inset of Figure 5(b)) corresponds to Au nanoplate viewed along $\langle 111\rangle$ direction and presents three sets of diffraction spots with six-fold rotational symmetry, which could be indexed to $\{220\},\{422\}$, and fractional $(1 / 3)\{422\}$ reflections. The SEAD is dominated by the kinematically forbidden fractional (1/3)\{422\} spots which are commonly observed in $\langle 111\rangle$ oriented flat-lying nanoplates. Similar forbidden reflections have been observed previously on thin films or platelets of $f c c$ structure crystal with $\{111\}$ surfaces and rather small thicknesses in the perpendicular 


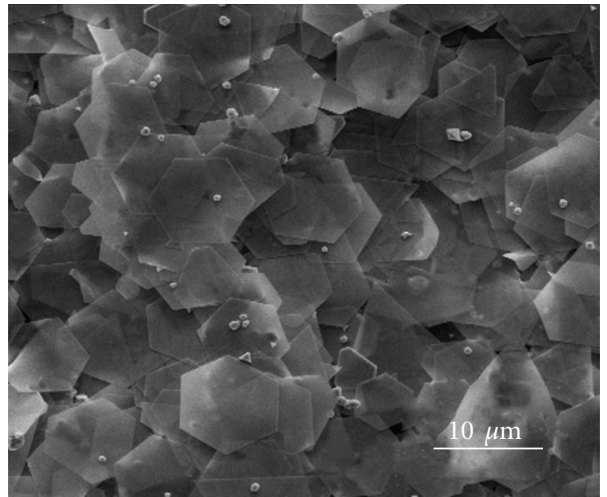

(a)

(c)

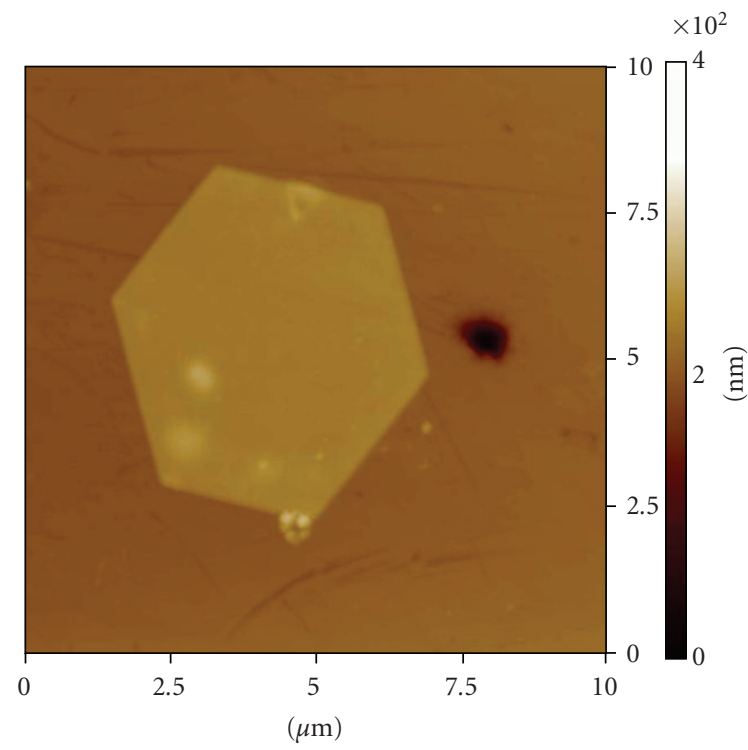

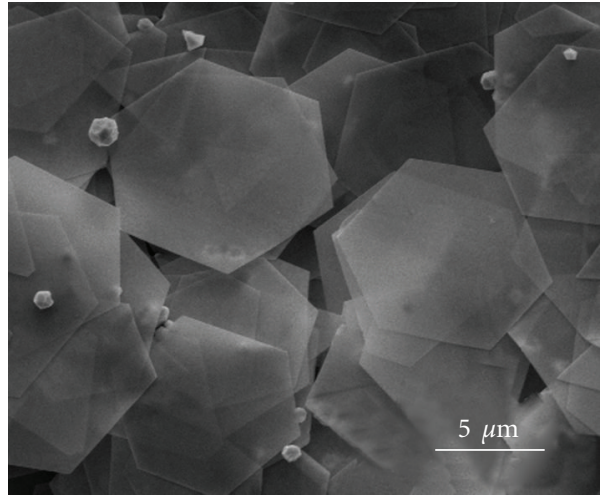

(b)

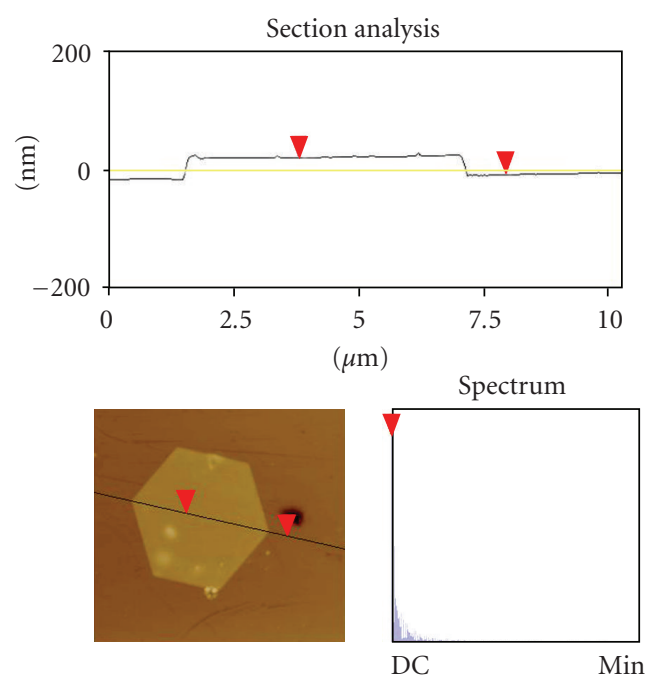

(d)

Figure 3: (a) Low- and (b) high-magnification FE-SEM images of obtained Au nanoplates deposited on a copper sheet. (c) and (d): AFM image and height profile of one single Au nanoplate.

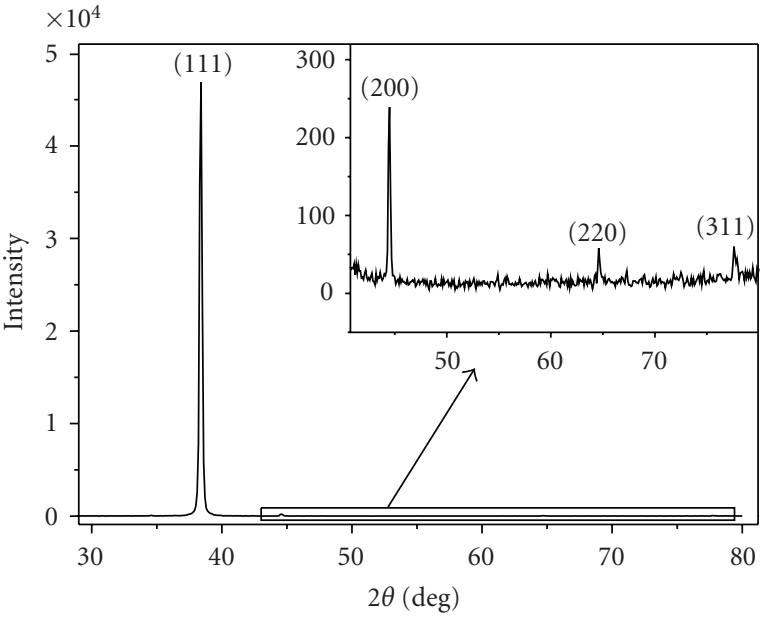

FIGURE 4: XRD pattern of the obtained Au nanoplates deposited on a glass slide. direction [37-41]. The (111) stacking fault lying parallel to the (111) surface is ever proposed to explain the occurrence of the $(1 / 3)\{422\}$ reflections [42-44]. Shown in Figure 5(c) is the HRTEM image of the edge part of one hexagonal Au nanoplate. The lattice spacing $(0.25 \mathrm{~nm})$ agrees fairly well with $3 \times\{422\}$ lattice spacing $(0.249 \mathrm{~nm})$ of Au crystal. This observation is strongly consistent with the SAED analyses and also demonstrated that these $3 \times\{422\}$ fringes build a perfect lattice and extend across the entire nanoplates.

3.3. Growth Conditions for Au Nanoplates. For further understanding of the growth conditions of the Au nanoplates, the synthesis process was carried out by varying the reaction parameters. First, we prepared Au nanocrystals by changing the molar ratios of $[\mathrm{CTAB}]$ and $\left[\mathrm{HAuCl}_{4}\right]$ with the same $\mathrm{HAuCl}_{4}$ concentration. The molar ratio between 2 and 3 was the most time-saving and favorable for the synthesis of regular Au nanoplates. Figures 6(a)-6(c) show the FE-SEM 


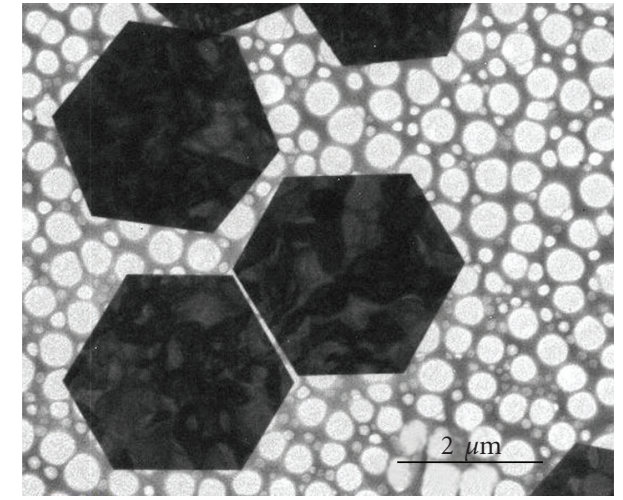

(a)

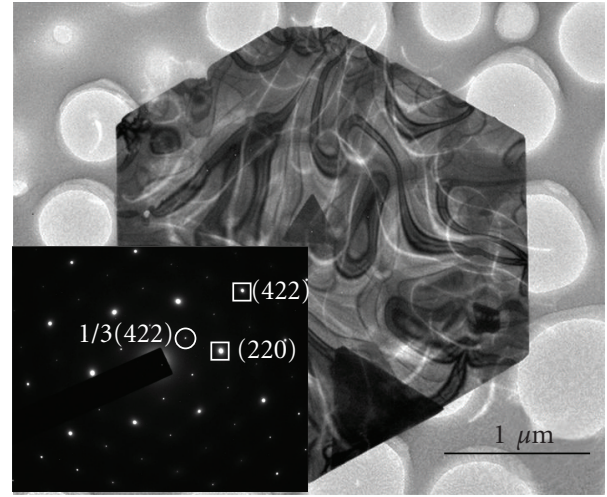

(b)

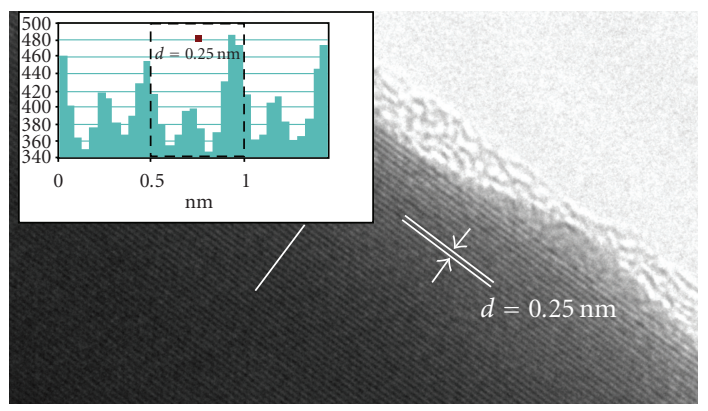

(c)

Figure 5: (a) TEM image of some Au nanoplates. (b) A typical TEM image of one hexagonal Au plate. The inset shows the corresponding SAED pattern taken by directing the electron beam perpendicular to the plate. The spots enclosed by squares could be indexed to the regular reflections $\{220\}$ and $\{422\}$. The spots enclosed by circles could be indexed to the fractional reflections $(1 / 3)\{422\}$. (c) HRTEM image of Au nanoplate. The measured plane spacing is $0.25 \mathrm{~nm}$, three times the $\{422\}$ lattice spacing.

images of Au nanoplates fabricated with the molar ratios of 5,3 , and 1 , respectively. With a molar ratio of 5 , the product is mainly composed of large Au plates with several tens of micrometers (Figure 6(a)). When the Au nanoplates were synthesized with a molar ratio of 3 , nanoplates of several micrometers in size are the main product, together with a few polyhedral Au particles (Figure 6(b)). Inserted in Figure 6(b) is the image of the boxed part, indicating that the (111) basal planes are perpendicular to the side facets. The sizes of $\mathrm{Au}$ nanoplates formed with a molar ratio of 1 are less than $5 \mu \mathrm{m}$ (Figure 6(c)). Figure 6(d) is the Au nanoplates synthesized without CTAB as will be described in the following. Figure 7 presents the times when the reaction system became colorless, that is, for completely reduction of $\mathrm{Au}(\mathrm{III})$ to $\mathrm{Au}(\mathrm{I})$, and completion of the synthesis. It can be seen that the reaction and the crystal growth can be completed within 30 minutes at the optimal molar ratio of 2 . When the molar ratio is lower or much higher than 2 , it decreases the reduction rate of $\mathrm{Au}(\mathrm{III})$ to $\mathrm{Au}(\mathrm{I})$ and hence nucleation efficiency of $\mathrm{Au}(0)$ into seeds in the solution. Accordingly, the reaction will last for a long time. These results clearly demonstrate that the CTAB concentration influences the shape and size of Au nanoplates and the molar ratio also plays an important role on the reduction rate.

When the synthesis was carried out in the absence of $\mathrm{CTAB}$ with $[\mathrm{PVP}] /\left[\mathrm{HAuCl}_{4}\right]$ molar ratio of 30 , the reaction and crystal growth would take a long time (more than 2 hours), and the main products were regular shapes (triangular, hexagonal, or truncated triangular) with edge length up to tens of micrometers, as shown in Figure 6(d). On the other hand, when only CTAB was applied in the synthesis system, the reaction lasted for a long time, in which the duration of colorless stage, that is, $\operatorname{Au}(\mathrm{I}) \rightarrow \operatorname{Au}(0)$, is more than 10 hours and only some particles with irregular shapes were found in the final product. Meanwhile, it is found that the reaction temperature is another important factor affecting the synthesis process and the shape of the final product. Thus, assisting with a certain concentrations of PVP and CTAB, temperatures among $140^{\circ} \mathrm{C} \sim 160^{\circ} \mathrm{C}$ are suitable for the formation of $\mathrm{Au}$ nanoplates. However, when the reaction temperature is at about $120^{\circ} \mathrm{C}$, the reaction process will slow down, and the colorless solution will be kept for more than 3 hours. The final product is dominated by a small number of large and thick Au plates (not shown).

From the above results, it can be seen that the presence and the concentration of PVP and CTAB in the growth solution played important roles for the time-saving synthesis of $\mathrm{Au}$ nanoplates. We found that the molar ratios of $[\mathrm{CTAB}] /\left[\mathrm{HAuCl}_{4}\right]=2$ and $[\mathrm{PVP}] /\left[\mathrm{HAuCl}_{4}\right]=30$ are optimal for the fabrication of regular hexagonal $\mathrm{Au}$ nanoplates. 


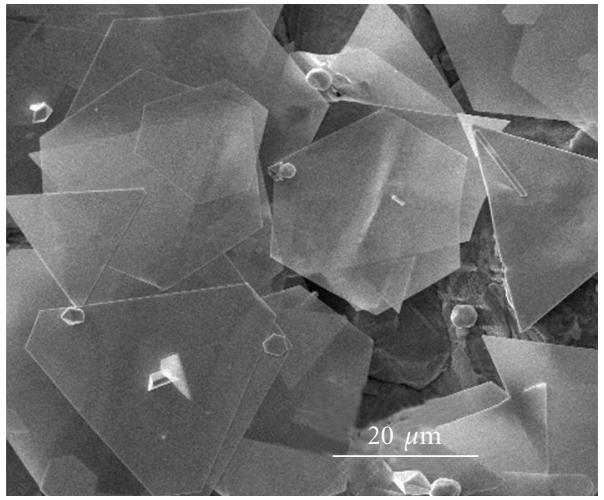

(a)

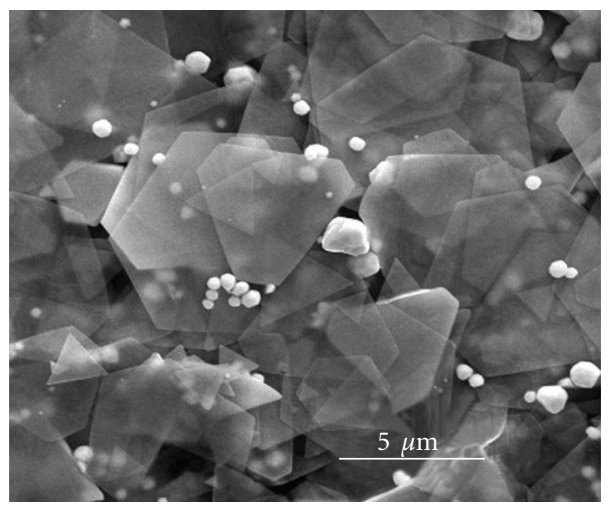

(c)

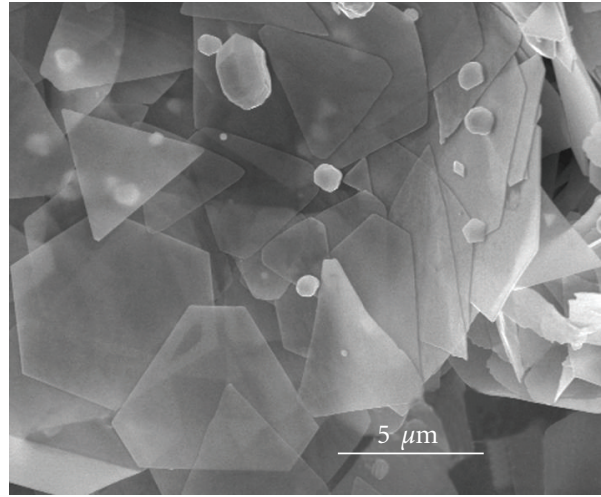

(b)

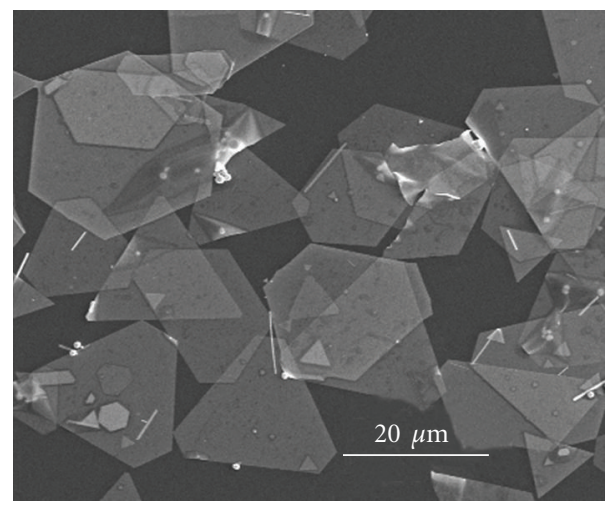

(d)

Figure 6: SEM image of Au plates obtained at $150^{\circ} \mathrm{C}$ with the different $[\mathrm{CTAB}] /\left[\mathrm{HAuCl}_{4}\right]$ molar ratios of: (a) 5, (b) 3, and (c) 1 . (d) Au plates obtained with the $[\mathrm{PVP}] /\left[\mathrm{HAuCl}_{4}\right]$ molar ratio of 30 and absence of CTAB. Inserted in (b) is a magnification image for the edge facet of box part, indicating the basal planes are perpendicular to the side facets.

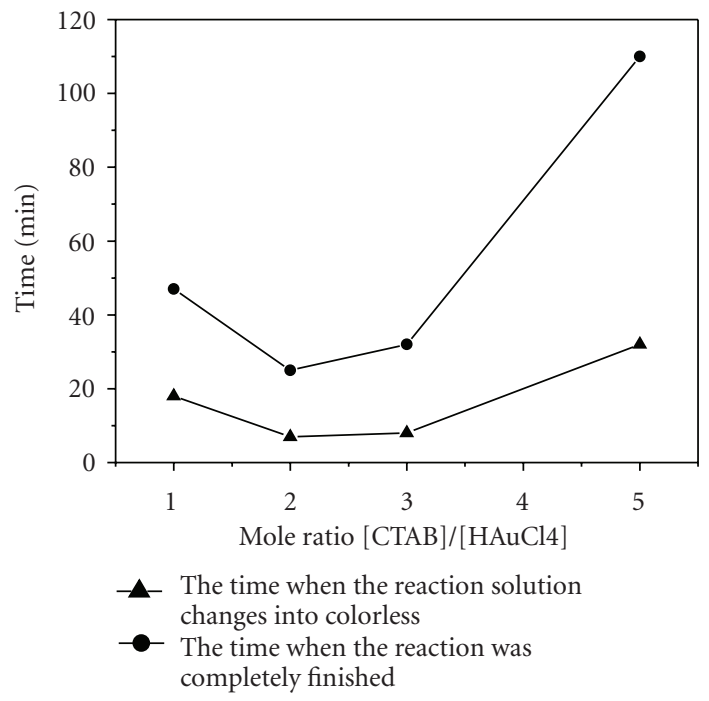

Figure 7: Plots of reaction time and different $[\mathrm{CTAB}] /\left[\mathrm{HAuCl}_{4}\right]$ molar ratios.

3.4. Proposed Mechanism on the Formation of Au Nanoplates. Although some groups have presented the possible growth mechanisms of the regular structure according to their results and fabricating conditions, the exact growth has yet to be established. Thus, it is believed that hexagonal-close-packed (hcp) structure and planar defects (e.g., twin defects or stacking faults) on the lateral facet of seeds play important roles in the growth orientation and final shape of the crystal $[43,45]$. Even for the mechanism itself, arguments on it were more proposed recently $[23,46]$. One is that twinned crystal seeds have not been observed to form in situ at present because of the fast nucleation and the limitation of measurement technique. From experimental results, we present our understanding on the nuclei formation and crystal growth behind anisotropic Au nanoplates.

3.4.1. Reduction of $A u(I I I)$. Previous studies revealed that surfactant CTAB could act as stabilizer and shape controller for the formation of anisotropic $\mathrm{Au}$ structures [23, 30, 35]. In our experiment, when the mixture of $\mathrm{HAuCl}_{4}$ and CTAB was kept at $80^{\circ} \mathrm{C}$, the color turned from yellow to red brown, indicating the formation of $\mathrm{AuBr}_{4}{ }^{-}$ions instead of $\mathrm{AuCl}_{4}{ }^{-}$ions. This is because $\mathrm{Br}^{-}$ions, a stronger complexation agent for $\mathrm{Au}(\mathrm{III})$ than $\mathrm{Cl}^{-}$ions, can effectively be stabilized by complexation with $\mathrm{Au}$ (III) ions leading to the formation of $\mathrm{AuBr}_{4}{ }^{-}$ions. With the presence $\mathrm{Br}^{-}$in 


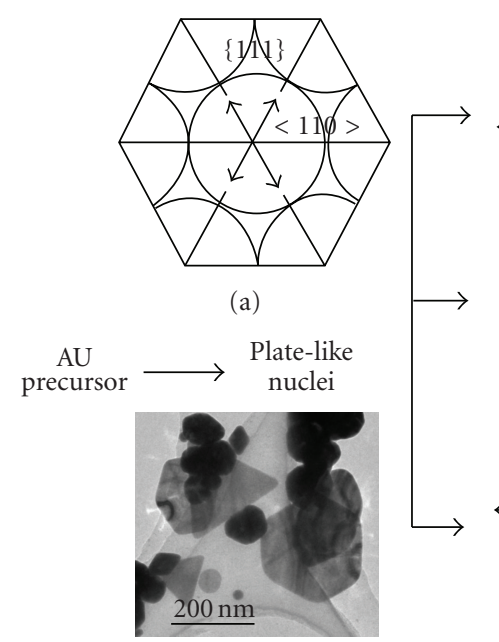

(d)
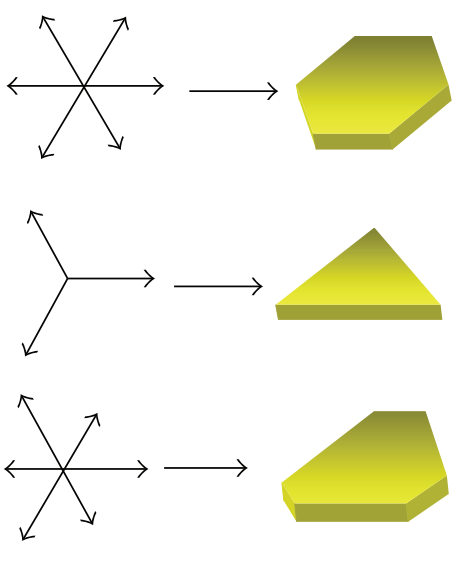

(b)

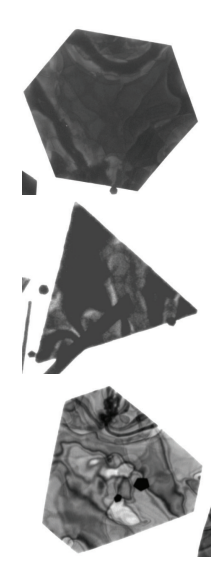

(c)

FIGURE 8: Proposed growth mechanisms and schematic illustration for the formation of Au nanoplates: (a) atoms arrangement of fcc \{111\} plane, (b) the formation of platy shapes along different $\langle 110\rangle$ directions, (c) the corresponding TEM images, and (d) TEM image of Au crystal sampled at initial growth stage.

$\mathrm{CTAB}$, the Au complexes $\left(\mathrm{CTA}^{+} \mathrm{AuBr}_{4}{ }^{-}\right)$were formed rather than formation of $\mathrm{Au}$ colloids [32]. At high temperature, the molecule of EG may be decomposed to a kind of reducing agent glycollic aldehyde with two aldehyde groups $(-\mathrm{CHO})$ that have strong reductive property. On the other hand, it is known that the polar pyrrolidone rings of PVP have affinity toward many chemicals to form coordinative compounds, in which metal ions would receive lone pair of electrons from the ligand of $-\mathrm{N}$ and $\mathrm{C}=\mathrm{O}$ in pyrrolidone ring [27]. Therefore, the solution rapidly became colorless at a high temperature $\left(150^{\circ} \mathrm{C}\right)$, implying the formation of colorless $\mathrm{AuBr}_{2}{ }^{-}$compound. Subsequently, the metastable state of $\mathrm{AuBr}_{2}{ }^{-}$was reduced to $\mathrm{Au}(0)$ instantaneously. The high reduction rate of $\mathrm{Au}(\mathrm{III})$ to $\mathrm{Au}(0)$ induced the supersaturation of $\mathrm{Au}(0)$ in solution, which in turn leads to the formation of Au nuclei.

3.4.2. Formation of Seeds and Crystal Growth. Under thermal equilibrium, twinned and multiply (usually five-fold) twinned particles are widespread habits both in synthetic and natural materials. However, the defect energy that exists along the twinned facets will limit the growth of twinned structure. This indicates that twinned seeds need to be confined to relatively small sizes. If these seeds expand in size rapidly, low surface energy of $\{111\}$ facets can no longer remedy the excessive energy, resulting in their transformation into single crystals [47]. Thus, when the reduction of metal ions to atoms is fast, single-crystal seeds will prevail over multiply twinned counterparts. On the other hand, in the hot system, the formed Au nuclei are exposed to oxygen, and the twinned seeds will be oxidized and dissolved in solution possibly due to the critical oxidation size, the oxidizability of reaction environment, or the instability of ultra fine particles $[48,49]$.
With the use of capping agent in the synthesizing system, the growth rates of crystal are often controlled (kinetically rather than thermodynamically) by the sticking probability on a given face $[23,50]$. The competition (and/or cooperation) between the surface energy difference and the coordination effect will confer the tendency to form single-crystalline nuclei bounded by a certain facet, which in turn not only determine the crystallographic growth of the particles but also drastically change the kinetics of crystal growth. At the favorite $[\mathrm{CTAB}] /\left[\mathrm{HAuCl}_{4}\right]$ and $[\mathrm{PVP}] /\left[\mathrm{HAuCl}_{4}\right]$ molar ratios, we suggest, though the exact interaction is still under study, that the polar groups are preferentially adsorbed on the sites of the $\{111\}$ planes of $\mathrm{Au}$ nuclei, which greatly decreases the surface energy of the $\{111\}$ planes and leads to preferential growth within the $\{111\}$ planes into two-dimensional structures. Then the question is: how the Au crystals grow into nanoplates with different shapes?

From our result, such as the perpendicular relation between the side facets and the basal planes (Figure 6(b)), the formation of Au nanoplates can be understood based on the growth of the (111) planes (Figure 8). From the data in the Figure 8(a), it can be seen that the nuclei grow uniformly along six $\langle 110\rangle$ directions if the growth environment is uniform, resulting in the formation of hexagonal plate surrounded by three pairs of $\{110\}$ lateral faces. However, in the open experiment system, the crystal growth would inevitably deviate from uniform growth along six $\langle 110\rangle$ directions. Therefore, truncated triangular and triangular plates will be formed if the nuclei are grown along different $\langle 110\rangle$ directions (or to some extent), as shown in Figure 8(b). Corresponding to schematic drawing, Figure 8(c) presents some typical TEM images of $\mathrm{Au}$ nanoplates observed in our experiment. For Au crystal sampled at initial growth stage, small $\mathrm{Au}$ nanostructures with triangular, hexagonal 
shapes are observed, as shown in Figure $8(\mathrm{~d})$, indicating the formation of different platy Au crystal seeds for further growth.

\section{Conclusions}

In summary, this paper provides a novel solution-phase approach for fast fabrication of single-crystalline $\mathrm{Au}$ nanoplates with well-defined hexagonal shapes by a modified polyol process with binary surfactants. The reduction of $\mathrm{Au}(\mathrm{III})$ and crystal growth were studied by optical absorption spectrum and color changes observation. Structural characterizations indicate that the obtained Au nanoplates, with (111) planes as two basal surfaces, have quite smooth surfaces in width and tens of nanometers in average thickness. The binary surfactants of PVP and CTAB with optimal concentration play a key role for promoting reduction of $\mathrm{Au}(\mathrm{III})$ to $\mathrm{Au}(0)$ and the formation of hexagonal $\mathrm{Au}$ nanoplates with nearly uniform size. The growth of the (111) planes in an open experiment system was proposed to explain the differences in shapes. This paper provides a highyield and time saving method for fabricating well-defined single-crystalline metal nanoplates.

\section{Acknowledgments}

This paper was financially supported by the national natural science foundation of China (no. 10704038 and no. 10772084). The authors thank Dr. Zhaosheng Li of Nanjing University for his help on the XRD and FE-SEM measurements.

\section{References}

[1] R. Jin, Y. Cao, C. A. Mirkin, K. L. Kelly, G. C. Schatz, and J. G. Zheng, "Photoinduced conversion of silver nanospheres to nanoprisms," Science, vol. 294, no. 5548, pp. 1901-1903, 2001.

[2] D. Astruc, F. Lu, and J. R. Aranzaes, "Nanoparticles as recyclable catalysts: the frontier between homogeneous and heterogeneous catalysis," Angewandte Chemie-International Edition, vol. 44, no. 48, pp. 7852-7872, 2005.

[3] D. S. Seferos, D. A. Giljohann, H. D. Hill, A. E. Prigodich, and C. A. Mirkin, "Nano-flares: probes for transfection and mRNA detection in living cells," Journal of the American Chemical Society, vol. 129, no. 50, pp. 15477-15479, 2007.

[4] S. E. Skrabalak, J. Chen, L. Au, X. Lu, X. Li, and Y. Xia, "Gold nanocages for biomedical applications," Advanced Materials, vol. 19, no. 20, pp. 3177-3184, 2007.

[5] A. Wijaya and K. Hamad-Schifferli, "Ligand customization and DNA functionalization of gold nanorods via round-trip phase transfer ligand exchange," Langmuir, vol. 24, no. 18, pp. 9966-9969, 2008.

[6] X. Huang, I. H. El-Sayed, W. Qian, and M. A. El-Sayed, "Cancer cell imaging and photothermal therapy in the nearinfrared region by using gold nanorods," Journal of the American Chemical Society, vol. 128, no. 6, pp. 2115-2120, 2006.

[7] J. Pérez-Juste, I. Pastoriza-Santos, L. M. Liz-Marzán, and P. Mulvaney, "Gold nanorods: synthesis, characterization and applications," Coordination Chemistry Reviews, vol. 249, no. 17-18, pp. 1870-1901, 2005.

[8] G. Wu, A. Mikhailovsky, H. A. Khant, C. Fu, W. Chiu, and J. A. Zasadzinski, "Remotely triggered liposome release by nearinfrared light absorption via hollow gold nanoshells," Journal of the American Chemical Society, vol. 130, no. 26, pp. 81758177, 2008.

[9] P. Zijlstra, J. W. M. Chon, and M. Gu, "Five-dimensional optical recording mediated by surface plasmons in gold nanorods," Nature, vol. 459, no. 7245, pp. 410-413, 2009.

[10] C. J. Murphy and N. R. Jana, "Controlling the aspect ratio of inorganic nanorods and nanowires," Advanced Materials, vol. 14, no. 1, pp. 80-82, 2002.

[11] J.-Q. Hu, Q. Chen, Z.-X. Xie, et al., "A Simple and effective route for the synthesis of crystalline silver nanorods and nanowires," Advanced Functional Materials, vol. 14, no. 2, pp. 183-189, 2004.

[12] T. K. Sau and C. J. Murphy, "Self-assembly patterns formed upon solvent evaporation of aqueous cetyltrimethylammonium bromide-coated gold nanoparticles of various shapes," Langmuir, vol. 21, no. 7, pp. 2923-2929, 2005.

[13] T. H. Ha, H.-J. Koo, and B. H. Chung, "Shape-controlled syntheses of gold nanoprisms and nanorods influenced by specific adsorption of halide ions," Journal of Physical Chemistry C, vol. 111, no. 3, pp. 1123-1130, 2007.

[14] S. Guo, S. Dong, and E. Wang, "Rectangular silver nanorods: controlled preparation, liquid-liquid interface assembly, and application in surface-enhanced raman scattering," Crystal Growth and Design, vol. 9, no. 1, pp. 372-377, 2009.

[15] C.-X. Kan, J.-J. Zhu, and X.-G. Zhu, "Silver nanostructures with well-controlled shapes: synthesis, characterization and growth mechanisms," Journal of Physics D, vol. 41, no. 15, Article ID 155304, 9 pages, 2008.

[16] Y. Xia, P. Yang, Y. Sun, et al., "One-dimensional nanostructures: synthesis, characterization, and applications," Advanced Materials, vol. 15, no. 5, pp. 353-389, 2003.

[17] C. Kan, X. Zhu, and G. Wang, "Single-crystalline gold microplates: synthesis, characterization, and thermal stability," Journal of Physical Chemistry B, vol. 110, no. 10, pp. 46514656, 2006.

[18] M. Tsuji, M. Hashimoto, Y. Nishizawa, M. Kubokawa, and T. Tsuji, "Microwave-assisted synthesis of metallic nanostructures in solution," Chemistry, vol. 11, no. 2, pp. 440-452, 2005.

[19] J. E. Millstone, G. S. Métraux, and C. A. Mirkin, "Controlling the edge length of gold nanoprisms via a seed-mediated approach," Advanced Functional Materials, vol. 16, no. 9, pp. 1209-1214, 2006.

[20] F. Kim, S. Connor, H. Song, T. Kuykendall, and P. Yang, "Platonic gold nanocrystals," Angewandte Chemie, vol. 43, no. 28, pp. 3673-3677, 2004.

[21] S. E. Skrabalak, B. J. Wiley, M. Kim, E. V. Formo, and Y. Xia, "On the polyol synthesis of silver nanostructures: glycolaldehyde as a reducing agent," Nano Letters, vol. 8, no. 7, pp. 2077-2081, 2008.

[22] J. E. Millstone, S. J. Hurst, G. S. Métraux, J. I. Cutler, and C. A. Mirkin, "Colloidal gold and silver triangular nanoprisms," Small, vol. 5, no. 6, pp. 646-664, 2009.

[23] Y. Xia, Y. Xiong, B. Lim, and S. E. Skrabalak, "Shapecontrolled synthesis of metal nanocrystals: simple chemistry meets complex physics?" Angewandte Chemie International Edition, vol. 48, no. 1, pp. 60-103, 2009.

[24] M. Maillard, P. Huang, and L. Brus, "Silver nanodisk growth by surface plasmon enhanced photoreduction of adsorbed $\left[\mathrm{Ag}^{+}\right]$," Nano Letters, vol. 3, no. 11, pp. 1611-1615, 2003. 
[25] C. C. Li, W. P. Cai, Y. Li, J. Hu, and P. Liu, "Ultrasonically induced Au nanoprisms and their size manipulation based on aging," Journal of Physical Chemistry B, vol. 110, no. 4, pp. 1546-1552, 2006.

[26] S. H. Chen and D. L. Carroll, "Silver nanoplates: size control in two dimensions and formation mechanisms," Journal of Physical Chemistry B, vol. 108, no. 18, pp. 5500-5506, 2004.

[27] C. X. Kan, W. P. Cai, C. C. Li, and L. Zhang, "Optical studies of polyvinylpyrrolidone reduction effect on free and complex metal ions," Journal of Materials Research, vol. 20, no. 2, pp. 320-324, 2005.

[28] Y. J. Xiong, J. Y. Chen, B. Wiley, Y. N. Xia, S. Aloni, and Y. Yin, "Understanding the role of oxidative etching in the polyol synthesis of Pd nanoparticles with uniform shape and size," Journal of the American Chemical Society, vol. 127, no. 20, pp. 7332-7333, 2005.

[29] Y. J. Xiong, J. Y. Chen, B. Wiley, Y. N. Xia, Y. Yin, and Z.-Y. Li, "Size-dependence of surface plasmon resonance and oxidation for Pd nanocubes synthesized via a seed etching process," Nano Letters, vol. 5, no. 7, pp. 1237-1242, 2005.

[30] Y. Z. Huang, W. Z. Wang, H. Liang, and H. X. Xu, "Surfactantpromoted reductive synthesis of shape-controlled gold nanostructures," Crystal Growth and Design, vol. 9, no. 2, pp. 858$862,2009$.

[31] A. A. Umar, M. Oyama, M. M. Salleh, and B. Y. Majlis, "Formation of high-yield gold nanoplates on the surface: effective two-dimensional crystal growth of nanoseed in the presence of poly(vinylpyrrolidone) and cetyltrimethylammonium bromide," Crystal Growth and Design, vol. 9, no. 6, pp. 2835-2840, 2009.

[32] T. Mortier, T. Verbiest, and A. Persoons, "Laser ablation of gold in chloroform solutions of cetyltrimethylammoniumbromide," Chemical Physics Letters, vol. 382, no. 5-6, pp. 650-653, 2003.

[33] J. E. Millstone, S. Park, K. L. Shuford, L. Qin, G. C. Schatz, and C. A. Mirkin, "Observation of a quadrupole plasmon mode for a colloidal solution of gold nanoprisms," Journal of the American Chemical Society, vol. 127, no. 15, pp. 5312-5313, 2005.

[34] L. Wang, X. Chen, J. Zhan, et al., "Synthesis of gold nano- and microplates in hexagonal liquid crystals," Journal of Physical Chemistry B, vol. 109, no. 8, pp. 3189-3194, 2005.

[35] H.-C. Chu, C.-H. Kuo, and M. H. Huang, "Thermal aqueous solution approach for the synthesis of triangular and hexagonal gold nanoplates with three different size ranges," Inorganic Chemistry, vol. 45, no. 2, pp. 808-813, 2006.

[36] Z. L. Wang, "Transmission electron microscopy of shapecontrolled nanocrystals and their assemblies," Journal of Physical Chemistry B, vol. 104, no. 6, pp. 1153-1175, 2000.

[37] X. Sun, S. Dong, and E. Wang, "High-yield synthesis of large single-crystalline gold nanoplates through a polyamine process," Langmuir, vol. 21, no. 10, pp. 4710-4712, 2005.

[38] X. P. Sun, S. J. Dong, and E. K. Wang, "Large-scale synthesis of micrometer-scale single-crystalline Au plates of nanometer thickness by a wet-chemical route," Angewandte Chemie International Edition, vol. 43, no. 46, pp. 6360-6363, 2004.

[39] Z. H. Li, Z. M. Liu, J. Zhang, et al., "Synthesis of singlecrystal gold nanosheets of large size in ionic liquids," Journal of Physical Chemistry B, vol. 109, no. 30, pp. 14445-14448, 2005.

[40] L.-P. Jiang, S. Xu, J.-M. Zhu, J.-R. Zhang, J.-J. Zhu, and H.-Y. Chen, "Ultrasonic-assisted synthesis of monodisperse singlecrystalline silver nanoplates and gold nanorings," Inorganic Chemistry, vol. 43, no. 19, pp. 5877-5883, 2004.
[41] B. Lim, P. H. C. Camargo, and Y. Xia, "Mechanistic study of the synthesis of Au nanotadpoles, nanokites, and microplates by reducing aqueous HAuCl4 with poly(vinyl pyrrolidone)," Langmuir, vol. 24, no. 18, pp. 10437-10442, 2008.

[42] A. I. Kirkland, D. A. Jefferson, D. G Duff, et al., "Structural studies of trigonal lamellar particles of gold and silver," Proceedings of the Royal Society A, vol. 440, no. 1910, pp. 589609, 1993.

[43] V. Germain, J. Li, D. Ingert, Z. L. Wang, and M. P. Pileni, "Stacking faults in formation of silver nanodisks," Journal of Physical Chemistry B, vol. 107, no. 34, pp. 8717-8720, 2003.

[44] A. Courty, A.-I. Henry, N. Goubet, and M.-P. Pileni, "Large triangular single crystals formed bymild annealing of selforganized silver nanocrystals," Nature Materials, vol. 6, no. 11, pp. 900-907, 2007.

[45] C. Lofton and W. Sigmund, "Mechanisms controlling crystal habits of gold and silver colloids," Advanced Functional Materials, vol. 15, no. 7, pp. 1197-1208, 2005.

[46] D. Aherne, D. M. Ledwith, M. Gara, and J. M. Kelly, "Optical properties and growth aspects of silver nanoprisms produced by a highly reproducible and rapid synthesis at room temperature," Advanced Functional Materials, vol. 18, no. 14, pp. 2005-2016, 2008.

[47] Y. J. Xiong, H. G. Cai, Y. D. Yin, and Y. N. Xia, "Synthesis and characterization of fivefold twinned nanorods and right bipyramids of palladium," Chemical Physics Letters, vol. 440, no. 4-6, pp. 273-278, 2007.

[48] H. Bi, W. Cai, C. Kan, L. Zhang, D. Martin, and F. Träger, "Optical study of redox process of Ag nanoparticles at high temperature," Journal of Applied Physics, vol. 92, no. 12, pp. 7491-7497, 2002.

[49] S. H. Im, Y.T. Lee, B. Wiley, and Y. Xia, "Large-scale synthesis of silver nanocubes: the role of $\mathrm{HCl}$ in promoting cube perfection and monodispersity," Angewandte ChemieInternational Edition, vol. 44, no. 14, pp. 2154-2157, 2005.

[50] D. K. Smith and B. A. Korgel, "The importance of the CTAB surfactant on the colloidal seed-mediated synthesis of gold nanorods," Langmuir, vol. 24, no. 3, pp. 644-649, 2008. 

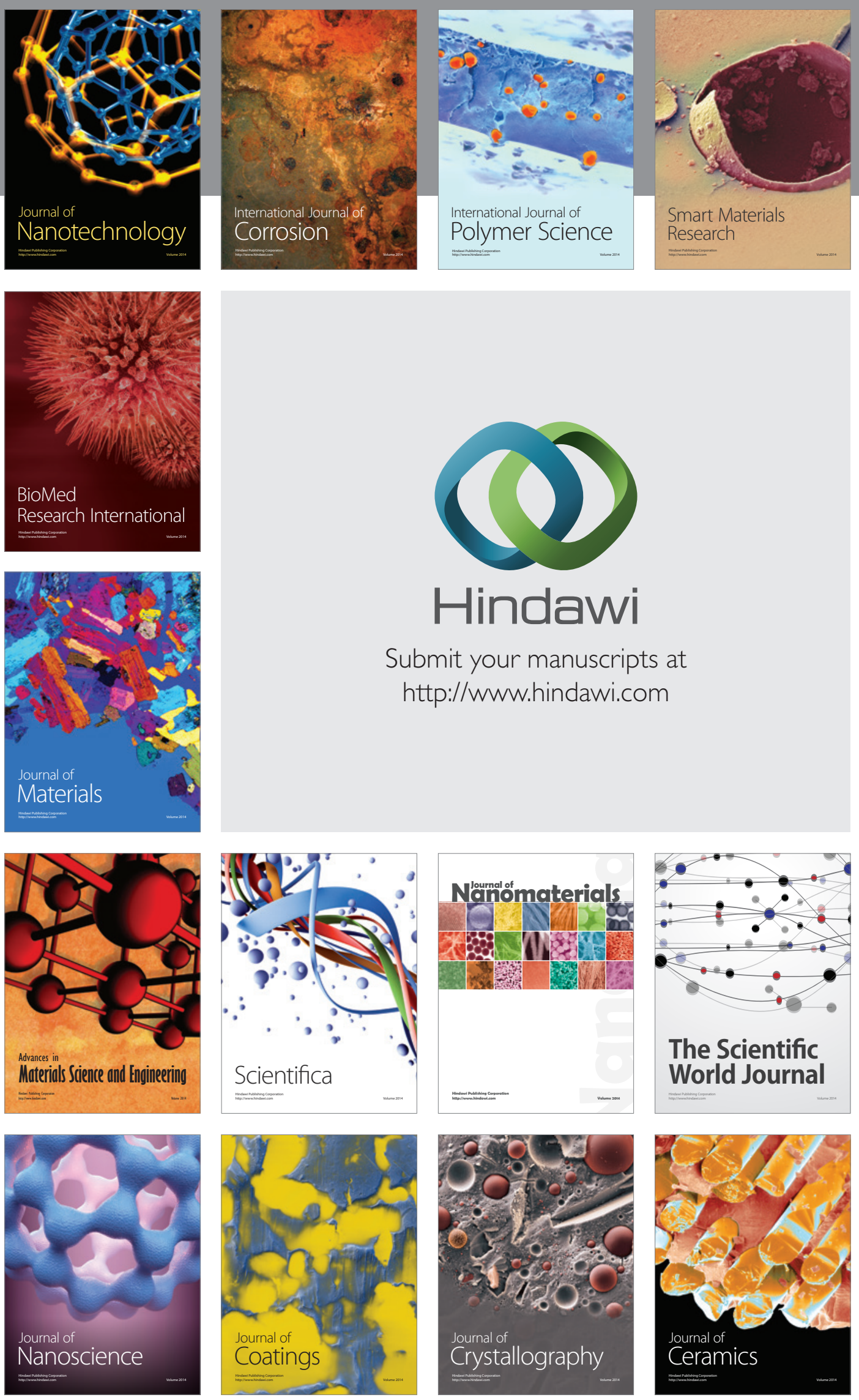

The Scientific World Journal

Submit your manuscripts at

http://www.hindawi.com

\section{World Journal}

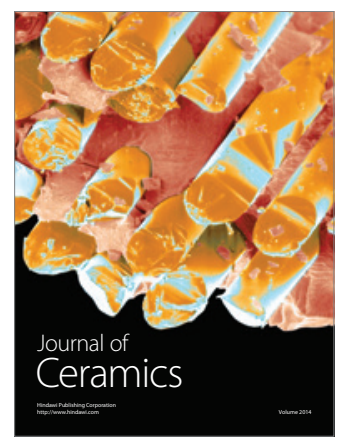

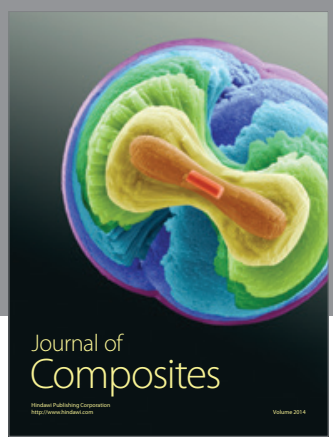
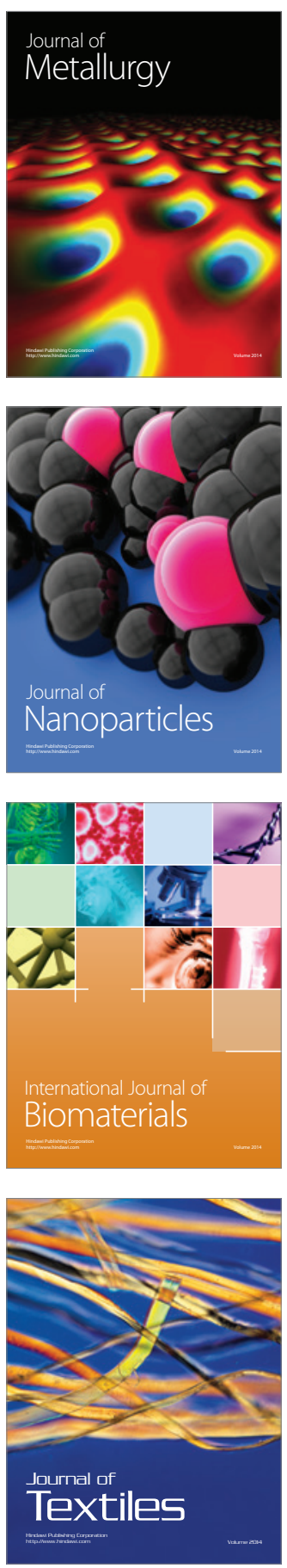